\title{
Atom Detection in Time-resolved TEM Image Series: Application of Computer Vision Techniques to Noise-degraded Frames
}

Ramon Manzorro ${ }^{1}$, Yuchen $\mathrm{Xu}^{2}$, Joshua Vincent ${ }^{1}$, Roberto Rivera ${ }^{3}$, David Matteson ${ }^{2}$ and Peter Crozier ${ }^{4}$

${ }^{1}$ Arizona State University, United States, ${ }^{2}$ Cornell University, United States, ${ }^{3}$ University of Puerto RicoMayaguez, United States, ${ }^{4}$ Arizona State University, Tempe, Arizona, United States

New advances in the field of aberration corrected in situ transmission electron microscopy (TEM) coupled with upgraded direct electron detectors provide atomic resolution information of materials exposed to chemical reaction conditions with spatial and temporal resolutions, respectively, of $0.1 \mathrm{~nm}$ and 10-3 s [1]. The atomic information encoding the evolution of the system is directly related to the coordinates of the columns and their intensity, related respectively to the position and occupancy of every column. However, high-speed acquisition times may imply a decrease in the number of counts per frame, thus leading to datasets severely degraded by noise. Consequently, accurately tracking the position and occupancy of atomic columns in time-resolved image series under such noisy conditions becomes a challenge, hindering the determination of dynamics (or so-called fluxional behavior) in evolving systems [2].

Conventional algorithms to record the motion of atomic columns and retrieve their intensity involve identifying local maxima and fitting a 2D Gaussian function over the region of interest [3,4]. Indeed, such algorithms output precise results when the signal-to-noise ratio (SNR) is high and the atomic column intensity follows a Gaussian distribution, providing coordinates and intensity accurately. However, TEM time-series acquired with high temporal resolution may suffer from low SNR, hindering the accuracy of the fitted coordinates and intensity. To address atom tracking in noisy data and overcome the uncertain output from the 2D Gaussian fitting method, we investigate an alternative, noise-robust blob detection technique for identifying objects in images that has been established in the field of computer vision.

A simulated time-series has been created by adding Poisson noise realizations to a single simulation of a $\mathrm{CeO} 2$ nanoparticle, leading to a 10-frame noisy image series where the ground truth, based on the information from the 3D structural model, provides an absolute reference value for coordinates and intensity. This simulated dataset allows a fair and objective comparison between different techniques. A blob detection algorithm [5] is applied herein for the first time to TEM images (up to our knowledge), and its performance is compared to a 2D Gaussian fitting algorithm. For this work, we use TRACT [4], a 2D Gaussian routine specifically designed for time-resolved phase contrast TEM image-series. Figure 1a illustrates one frame of the simulated nanoparticle after adding Poisson noise. Figures $1 \mathrm{~b}$ and $1 \mathrm{c}$ depict the fitted position and intensity from the Gaussian peak fitting and blob detection algorithms, respectively. As seen in comparing the two methods, the blob detection offers a more precise and more accurate fit on both the atomic column position and intensity. Given the promising results obtained from blob detection, the method has been applied to an experimental in situ TEM image series of a $\mathrm{CeO} 2$ nanoparticle undergoing dynamic structural behavior $[6,7]$. 
(a) $\mathrm{CeO}_{2}$ NOISY SIMULATED IMAGE

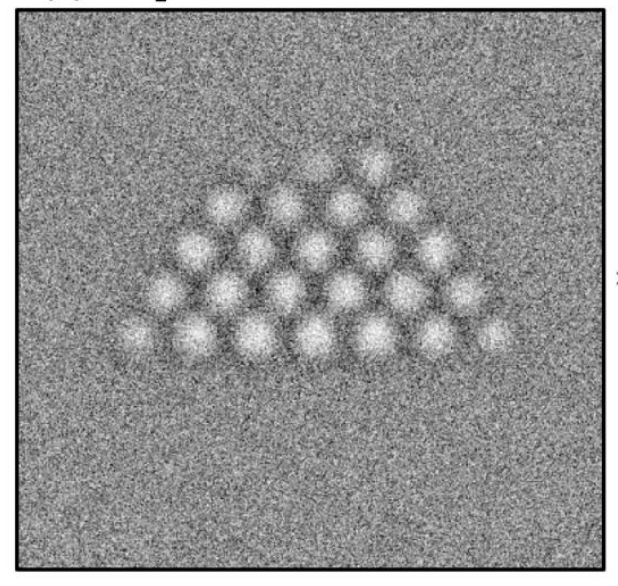

(b) TRACT (GAUSSIAN PEAK FITTING)

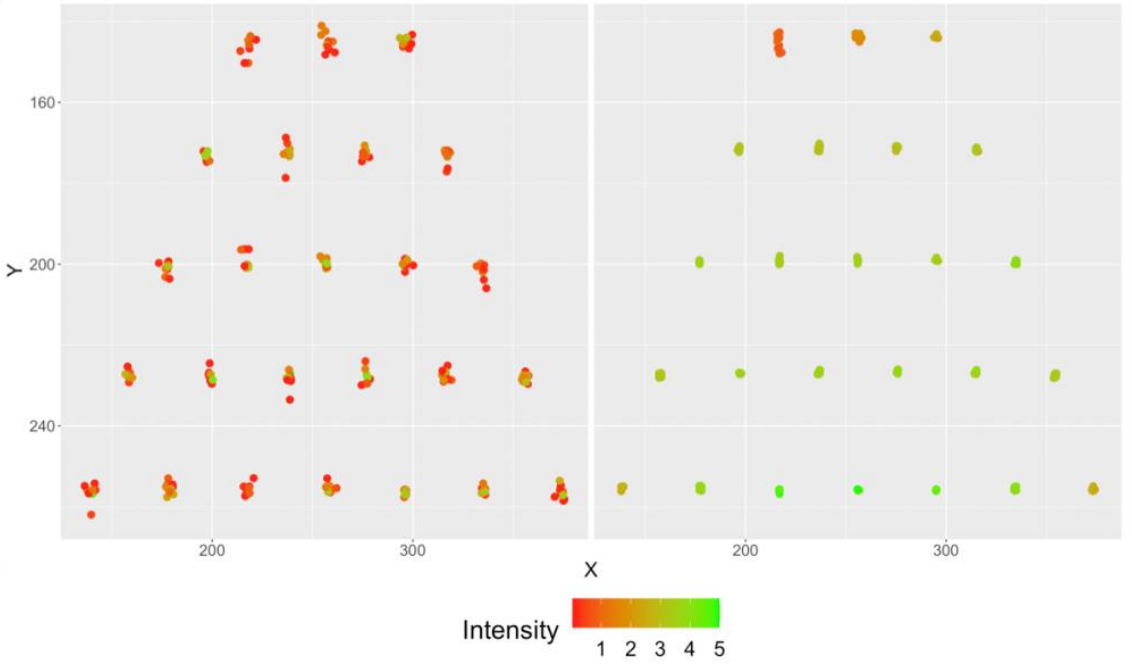

Figure 1. (a) Single frame from 10-frame simulated movie of a $\mathrm{CeO} 2$ nanoparticle after adding Poisson noise with a mean value in vacuum of 139 and standard deviation of 34.5, presenting at the top surface three columns with 1, 2 and 3 atoms (from left to right). (b) and (c) illustrate the performance over noisy simulated dataset of blob detection and Gaussian peak fitting respectively, showing the dispersion of the points the precision on the coordinates and the color dispersion the precision on the intensity, which have been normalized in the range $[0,5]$.

\section{References}

[1] Faruqi, A., et al. Nucl. Instrum. Methods Phys. Res, 2018. 878, p. 180-190.

[2] Lawrence, E. L., et al. Microscopy and Microanalysis, 2020. 26, p. 86-94.

[3] Nord, M., et al. Adv. Struct. Chem. Imaging, 2017. 3, p. 9-20.

[4] Levin, B. D. A., et al. Ultramicroscopy, 2019. 213, 112978.

[5] Lindeberg, T., et al. Image Vision Comput., 1997. 15, p. 415-434.

[6] Manzorro, R., et al., manuscript in preparation.

[7] We gratefully acknowledge support of NSF grant CBET-1604971, NRT-1922658, CCF-1934985, OAC-1940097, OAC-1940124 and OAC-1940276, and the facilities at ASU's John M. Cowley Center for High Resolution Electron Microscopy. 